\title{
Pulsed electropolymerization of PEDOT enabling controlled branching
}

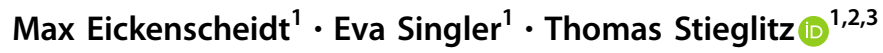

Received: 1 February 2019 / Revised: 18 April 2019 / Accepted: 10 May 2019 / Published online: 31 May 2019

(c) The Author(s) 2019. This article is published with open access

\begin{abstract}
Controlling the growth of conductive polymers via electrolysis enables defined surface modifications and can be used as a rapid prototyping process. In this study, the controlled dendritic growth of poly(3,4-ethylenedioxythiophene) in a twoelectrode setup was investigated by pulsed voltage-driven electropolymerization of the precursor EDOT and a low concentration of tetrabutylammonium perchlorate dissolved in acetonitrile. Rapid growth of different polymeric shapes was reliably achieved by varying the reduction voltage and duty factor. The obtained structures were optically examined and quantified using fractal dimensions. Their shapes ranged from solid coatings over branched fractals to straight fibers without requiring any template. These rapid and controllable electropolymerization processes were further combined to increase conductor complexity.
\end{abstract}

\section{Introduction}

Organic conductors are foreseen as the next generation of electronic and optical devices. Their adjustable mechanical and electrical properties (e.g., their flexibility and intrinsically large active surface) are unique features compared with those of established electronics. In particular, poly (3,4-ethylenedioxythiophene) (PEDOT), as a prominent representative of $\pi$-conjugated aromatic polymers, is widely used in many fields ranging from neuroscience [1-3] and surface modification [4] to solar technology [5]. Although the material properties and surfaces are mainly defined by the supporting salts or counterions, the outer shape is difficult to control without using templates. Recently, it has been shown that pulsed electropolymerization is a possible candidate to achieve another degree of freedom [6, 7]. A low-frequency zero-mean voltage was applied to gold wires, and strongly directed

Supplementary information The online version of this article (https:// doi.org/10.1038/s41428-019-0213-4) contains supplementary material, which is available to authorized users.

Max Eickenscheidt

eickenscheidt@imtek.de

1 Department of Microsystems Engineering (IMTEK), University of Freiburg, Freiburg, Germany growth was observed. A change in growth toward stronger branching was also achieved by using different counterions [6] or spatial limitation [8] of diffusion/migration. Motivated by these studies, we investigated the influence of voltage amplitudes other than zero-mean waveforms and the duty factor (DF) of low-frequency $5 \mathrm{~Hz}$ electropolymerization.

We hypothesized that the unidirectional strength of the electric field is not only critical for the electropolymerization process, as is known, for example, from insulation breakdowns forming electrical trees. We also contended that pure diffusion is not the only factor responsible for the formation of fractal structures, as is known from crystal formation, but the alternating depletion and accumulation of radical cations leading to PEDOT at the electrode interface are also ultimately controlling the final structure. Based on bipolar electropolymerization experiments, the electrolytic reaction system is simplified, and the charges for the pure oxidation of EDOT are provided directly by a metallic conductor. A very low concentration of the supporting salt is also chosen to obtain high mass transport owing to migration of the charged

2 BrainLinks-BrainTools, University of Freiburg, Freiburg, Germany

3 Bernstein Center Freiburg, University of Freiburg, Freiburg, Germany 
species. This is predicted by a theoretical description reported elsewhere using a Nernst-Planck-Poisson model without electroneutrality, which well describes the unique properties of such systems $[9,10]$. The anodic voltage for EDOT oxidation was thereby fixed to provide radical cations in the vicinity of the electrode, which transformed subsequently to a dication and further polymerized with conductive PEDOT structures. The duration of this anodic phase as well as the interlaced cathodic phases was varied using different negative voltages at a fixed frequency of 5 Hz. To the best of the authors' knowledge, this work is the first report of pulsed and asymmetric electropolymerization. Branching and growth dynamics were evaluated and classified, and the results are presented using a vivid model.

\section{Experimental procedure}

\section{Materials and Instrumentation}

The electropolymerization of PEDOT was performed in a solution containing $50 \mathrm{~mm}$ EDOT with $1 \mathrm{~mm}$ tetrabutylammonium perchlorate (TBAP) as the supporting salt and acetonitrile (ACN) as the solvent. All chemicals were obtained from SIGMA-ALDRICH and used without any modifications. As a counter electrode (CE), a large platinum (Pt) plate $\left(0.75 \mathrm{~cm}^{2}\right)$ was used (GoodFellow, Cambridge, $\mathrm{UK}$ ), whereas the working electrode (WE) was a gold (Au) wire that was $200 \mu \mathrm{m}$ in diameter and stripped at the tip (Polyfil AG). The two electrodes were immersed in a sealed beaker at a distance of $x_{0}=5 \mathrm{~mm}$ from each other unless otherwise mentioned (Fig. 1). During the experiments, the measuring chamber was covered to minimize the evaporation of ACN.

Open-circuit potential measurements were performed with an AutoLab spectrum analyzer (Autolab PGSTAT128N, Metrohm Autolab B.V., Utrecht, Netherland) prior to the start of the experiments. The determined value was used as a baseline for a periodic rectangular function with a fixed frequency of $5 \mathrm{~Hz}$, which was applied to the electrodes with an arbitrary waveform generator (Agilent Series 33500B, Keysight Technologies, Santa Rosa, California, USA). The maximum voltage $u_{\max }$ was kept constant at $4 \mathrm{~V}$, whereas the minimum voltage $u_{\min }$ and the DF were varied. The DF is hereby defined as the duration of the anodic pulse divided by the duration of the cathodic pulse per period (Fig. 1a). In each experiment, the waveform was applied for $120 \mathrm{~s}$, during which the growth behavior was optically recorded using a microscope camera (DigiMicro Scale 2.0, dnt GmbH, Dietzenbach, Germany) and the fine structures were resolved with a scanning

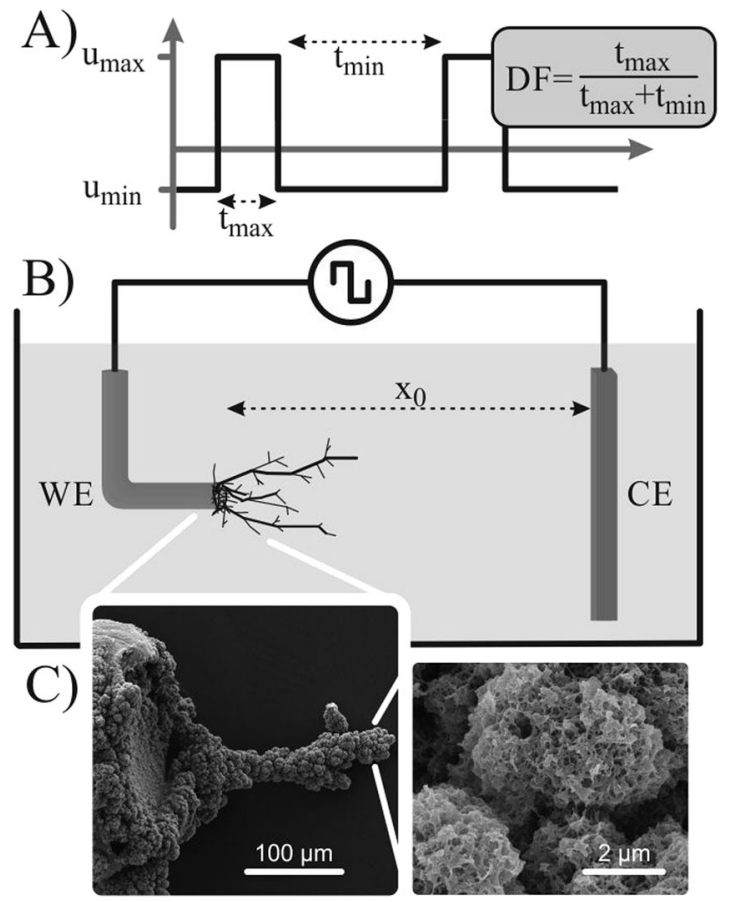

Fig. 1 Experimental setup. a Rectangular waveform with different voltage amplitudes $\left(u_{\max }\right.$ to $\left.u_{\min }\right)$ and duty factor DF were applied to two electrodes in a saturated EDOT solution. b Schematic illustration of the setup and PEDOT growing at the gold working electrode (WE) placed at a distance of $x_{0}$ from the platinum counter electrode (CE). c Scanning electrode microscopy image of the resulting PEDOT fibers (30 s of stimulation with $u_{\min }=-4 \mathrm{~V}$ and $\mathrm{DF}=75 \%$ ) showing initial polymerization near the electrode edge leading to a single polymeric strand

electron microscope (Nova NanoSEM 430, FEI, Hillsboro, Oregon, USA). All experiments were carried out at room temperature.

\section{Optical evaluation}

The growth velocity was measured by analyzing the video recordings, and a subsequent box-counting method was used to determine the fractal dimension by a linear approximation of 13 measured values $f_{\mathrm{dim}}=-\log (N) / \log$ $(\varepsilon)$, which provides information about the complexity or fractality of a system [11]. All calculations were performed with Fiji [12]/FracLac (BoxCounting), and 13 different sized boxes ( $\varepsilon=1-64$ pixels) were used to scan the number of boxes $(N)$ containing the PEDOT structure in a binary representation of the pictures. It should be noted that the analyses were carried out on a two-dimensional projection of the three-dimensional spatial structure. However, linear monotonic relationships between the 3D fractal dimension and its $2 \mathrm{D}$ projection have been previously suggested [13], which supports the proposed classification. 

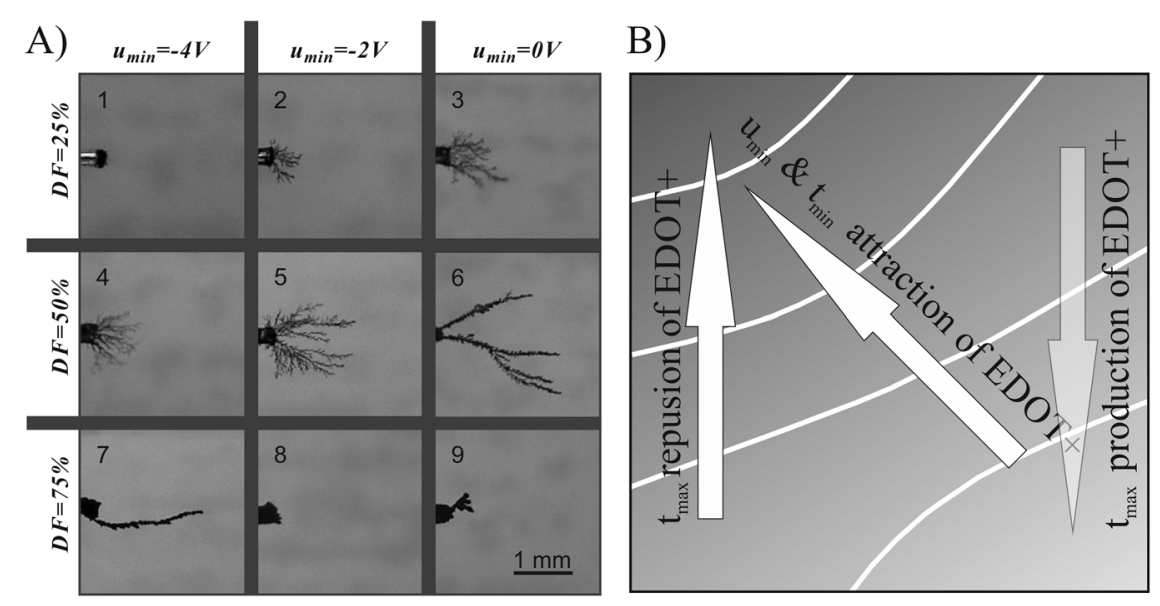

Fig. 2 a Growth behavior after $120 \mathrm{~s}$ of electropolymerization using a square wave with different duty factors $D F=t_{\max } /\left(t_{\max }+t_{\min }\right)$ and negative potentials $u_{\min }$ (positive amplitude is fixed to four volts). Pictures 3-7 show strong directional growth towards the counter electrode on the right-hand side but differ in branching (pictures are of

\section{Results and discussion}

\section{Waveform parameters}

Previous studies have demonstrated the possibility of obtaining conductive polymer fibers by electropolymerization via low-frequency alternating voltage electrolysis [6]. A low-frequency voltage was also applied in this work, whereby the focus was not on a wireless connection to the electrodes but rather on the exact manipulation of the stimulation parameters. In the two-electrode setup, reliable electropolymerization of EDOT was achieved at the gold WE with a fixed positive amplitude of four volts. During this process, clearly recognizable PEDOT structures formed at the tip of the WE, whereas no deposits were visible on the large platinum CE. By changing the negative amplitude $u_{\text {min }}$ and the DF of the rectangular signal, the growth velocity and the degree of branching were reliably controlled (Fig. 2a). Continuous electropolymerization for $120 \mathrm{~s}$ led to a significant difference in the final length, thickness, and complexity. A high degree of branching and therefore treelike growth were observed for DFs of $25 \%$ with small cathodic voltages $\left(u_{\min }=-2 \ldots 0 \mathrm{~V}\right)$ and $50 \%$ with larger repulsive phases $\left(u_{\min }=-4 \ldots-2 V\right)$ (Fig. 2a, panels 2-5). In the case of a weak cathodic phase owing to a short duration or a low negative potential (Fig. 2a, panels 6-7), nearly no degree of branching was observed, and the PEDOT directly grew to the CE. These structures reached a maximum length of $3.3 \mathrm{~mm}$, which corresponds to a maximal growth speed of $27 \mu \mathrm{m} / \mathrm{s}$. In addition to these stimulation parameters, film-like layers or solid volumes with minimal branching were formed on the gold electrode (Fig. 2a, panels 1, 8, and 9). It is assumed that migration had the same size). The proposed parametric influence on the concentration of EDOT + cations during pulsed electropolymerization at the phase boundary is shown in $\mathbf{b}$. Arrows point towards higher concentrations in close proximity to the electrode surface

a major role in structure formation, attracting, or repulsing all charged particles along the electric field. The electric field is given by the applied current density, which is highly inhomogeneous near the WE or the PEDOT structure, because of the large overpotential and the high-frequency components during switching between positive and negative voltage [14]. The highest field densities at high frequency were generated at the edges and tips. This behavior became evident when applying rectangular alternating voltages to an uncoated gold wire. A solid ring of PEDOT was formed in the first few seconds at the edges of the electrodes, regardless of the parameters used. This ring was the starting point for the spontaneous nucleation of the main branches (compare the SEM image in Fig. 1c). The initial branches then preferentially continued to grow out at a constant speed towards the CE (Table 1). Depending on the stimulation parameters, higher branching occurred, or side branches were discriminated against the main branch, and only spines were formed. In most cases, this propagation slightly decelerated after $100 \mathrm{~s}$. No differences in the final structure of the formed polymer were observed with different stimulation parameters-the porosity and the substructure of beads $5 \mu \mathrm{m}$ in diameter (Fig. 1c, right side) were comparable throughout the stimulation matrix.

The longer the positive phase was (larger DF), the more EDOT was oxidized. As a result, a back-creeping PEDOT layer around the wire insulation and a thicker general appearance of the grown structures were observable (see supplementary video). In particular, when DF was $75 \%$ and the cathodic phase was -2 or $0 \mathrm{~V}$, a large throughput of EDOT/PEDOT happened, leading to visible convection of the produced PEDOT in the shape of a black veil from the WE to the CE. Throughout the entire growth phase, the 
Table 1 The growth rate of the polymers analyzed between 20 and $120 \mathrm{~s}(N=3)$

\begin{tabular}{llll}
\hline Constant slope $(\mu \mathrm{m} / \mathrm{s})$ between 40 and $120 \mathrm{~s}$ process time & \\
\hline $\mathrm{DF}$ & $u_{\min }=-4 \mathrm{~V}$ & $u_{\min }=-2 \mathrm{~V}$ & $u_{\min }=0 \mathrm{~V}$ \\
\hline $25 \%$ & Length $0.25 \pm 0.10$ & Length: $4.40 \pm 0.83$ & Length: $7.31 \pm 1.46$ \\
& Height: $0.87 \pm 0.68$ & Height: $2.87 \pm 1.36$ & Height: $8.01 \pm 1.40$ \\
$50 \%$ & Length: $3.92 \pm 1.26$ & Length: $14.1 \pm 2.51$ & Length: $18.3 \pm 4.70$ \\
& Height: $5.02 \pm 2.32$ & Height: $8.69 \pm 3.47$ & Height: $3.46 \pm 3.10$ \\
$75 \%$ & Length: $14.8 \pm 0.36$ & Length: $1.53 \pm 1.20$ & Length: $1.64 \pm 1.01$ \\
& Height: $16.8 \pm 5.07$ & Height: $2.27 \pm 1.03$ & Height: $1.95 \pm 1.21$ \\
\hline
\end{tabular}

The lateral and horizontal extension were determined by measuring the maximum spread of all pictures in $20 \mathrm{~s}$ intervals during the electropolymerization
Table 2 Fractal dimension $f_{\text {dim }}$ for the respective growth pattern shown in Fig. 2a

\begin{tabular}{llll}
\hline $\mathrm{DF}$ & $u_{\min }=-4 \mathrm{~V}$ & $u_{\min }=-2 \mathrm{~V}$ & $u_{\min }=0 \mathrm{~V}$ \\
\hline $25 \%$ & $($ film $)$ & $($ group 1) & (group 2) \\
& $1.029 \pm 0.211$ & $1.403 \pm 0.033$ & $1.468 \pm 0.040$ \\
$50 \%$ & $($ group 1) & $($ group 2) & (group 3) \\
& $1.402 \pm 0.007$ & $1.461 \pm 0.043$ & $1.248 \pm 0.007$ \\
$75 \%$ & $($ group 3) & $($ film) & (film) \\
& $1.272 \pm 0.038$ & $1.027 \pm 0.219$ & $1.107 \pm 0.087$ \\
\hline
\end{tabular}

The standard deviation of several repetitions $(N=3)$ was considerably smaller than that between the different parameter sets. Similar fractals are clustered in subgroups

existing branches were mechanically stable without presenting broken structures. After switching off the alternating voltage, it was observed that the long thin structures bent slightly downwards following gravity.

Throughout several experiments, the constant growth rates and spatial distribution of the trees presented high variability (Table 1), but the growth behavior seemed to be similar. To quantify the growth pattern, the fractal dimen$\operatorname{sion} f_{\text {dim }}$ of the final structure was evaluated to measure the bifurcation. The gold wire and the backwards creeping PEDOT were not included in the analysis. Similar polymer structures resulted in similar $f_{\text {dim }}$ values; i.e., the spatial structure of the objects was nearly the same (Table 2). Three categories of different fractals were clearly distinguished, which was supported by visual inspection. Subgroup clustering was observed (panels 2/4, 3/5, 6/7 and 1/8/9 in Fig. 2a). The small standard deviation reflects the reliability of the process and the distinctness between the groups.

The similarity between the different panels of Fig. $2 b$ can be explained if one assumes that the supporting salt and the electroactive species do not undergo any reduction and that migration of EDOT + ions is the main factor. During the positive phase, EDOT is oxidized, and EDOT + is repulsed. A longer anodic phase (from top to bottom in the matrix) leads to a higher throughput, resulting in thicker and darker grown structures. Furthermore, a longer positive phase pushes more EDOT cations away from the direct vicinity of the electrode. This phenomenon results in a heavily doped PEDOT structure with only a few EDOT + ions in near proximity, decreasing with DF proportional to $t_{\max }$. The strength and duration of the negative pulse (larger $t_{\min }$ and/ or $u_{\text {min }}$ ) provide the initial attraction and number of EDOT cations, which are available. The concentration is also strongest near the edges and tips. Different patterns emerge after switching to the cathodic pulse. In the first column of the matrix, an attractive migration to the tips of the structure starts following the electric field lines (lower row), and with longer duration, positive ions come in close proximity to the entire PEDOT structure (upper row). In panel 1 of Fig. 2a, rather few monomers are oxidized, but they are all attracted and act as nuclei, resulting in a film rather than a tree-like structure. In panel 7, on the other hand, a large number of EDOT cations are formed and pushed away, and in the short negative phase, they are only attracted back to the tip of the electrode, resulting in directed growth. In contrast, no reducing voltage is applied in the third column of Fig. 2a. The instantaneous voltage change and the subsequently induced reorganization of the different ions near the electrode, however, also generate an inhomogeneous electric field resulting in higher migration at the tips (see panel 6). If the negative phase is applied longer (first row), there is general diffusion back to the whole electrode, and the formed PEDOT structures become bulkier. In the cases of panels 8 and 9, a large throughput of EDOT/PEDOT occurs; however, the strong repulsive forces generated by the long-lasting positive phase lead to visible convection of the produced PEDOT in the shape of a black veil (see supplementary video), and controlled outgrowth of PEDOT breaks down. The strengths (duration and amplitude) of the two phases with the attraction and repulsion of the formed EDOT cations thus seem to be two counteracting effects.

\section{Temporal and spatial variation}

The temporal evolution of the fractal dimension was, in all cases, asymptotic behavior towards the final value given in 
Fig. 3 Growth structures after $120 \mathrm{~s}$ of electropolymerization $\left(u_{\min }=-2 \mathrm{~V}, \mathrm{DF}=50 \%\right)$ while varying the anodic-cathodic electrode distance from $65 \mathrm{~mm}$ to $5 \mathrm{~mm}$ (fractal dimensions are $1.187,1.269,1.383$, and 1.502, respectively)

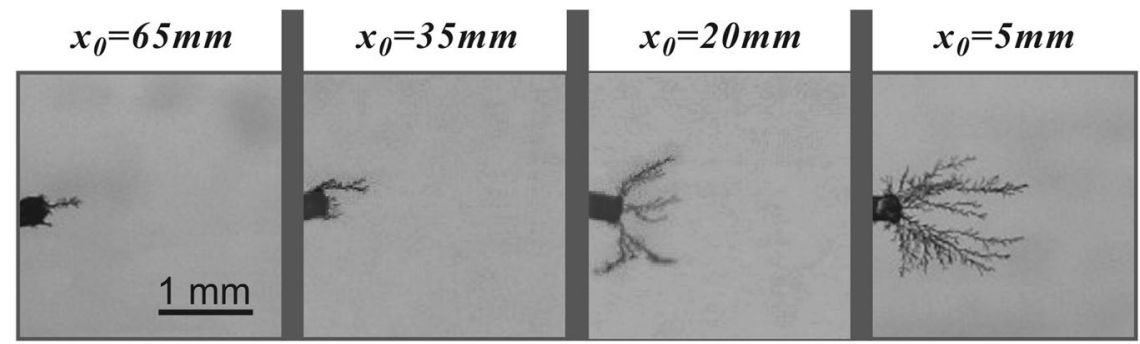

Table 2. Similar to the $f_{\text {dim }}$ values, the growth rates (Table 1) rose in the first $40 \mathrm{~s}$ and stabilized around the given value. In all experiments, this distance was fixed to $5 \mathrm{~mm}$, whereas in cases of high directional growth (panel 6-8 in Fig. 2a), a $2 \mathrm{~mm}$ distance was achieved after $2 \mathrm{~min}$ of electropolymerization. With ongoing polymerization, the decreasing distance between the working and CEs had no noticeable influence on either the $f_{\text {dim }}$ value or the growth rate.

On the other hand, an increase in the initial distance between the WE and the CE strongly affected the propagating fiber (Fig. 3). The growth rate and complexity of the grown structures (fractal dimension) decreased as the distance increased to $20 \mathrm{~mm}, 35 \mathrm{~mm}$, and $65 \mathrm{~mm}$. Furthermore, the growth rate and the final volume of the polymeric structures were lower with a greater distance. Even the back-creeping PEDOT along the insulation was decreased to a lesser extent. As the distance between the electrodes increased, a higher proportion of the voltage dropped across the electrolyte. Nonetheless, with an electrode diameter of $200 \mu \mathrm{m}$ and distances of $>5 \mathrm{~mm}$, no significant changes in the field or potential distributions were expected [15]. However, the access resistance of the electrode system shows a low distance dependence (Supplementary Fig. 1). It is doubtful whether this dependence would solely lead to the observed changes. The observed unidirectional convection (from the WE to the $\mathrm{CE}$ ) of the conductive oligomer and unbound polymers may have an additional influence.

\section{Two-step process}

The experiments showed that a reduction in the distance to below $10 \mathrm{~mm}$ did not lead to any obvious changes in the growth behavior. Therefore, in a further experiment, in addition to the linear growth of the PEDOT with parameter set $6\left(u_{\min }=0 \mathrm{~V}, \mathrm{DF}=50 \%\right)$, subsequent branching with parameter set $5\left(u_{\min }=-2 \mathrm{~V}, \mathrm{DF}=50 \%\right)$ was observed. Both stimulation pulses were applied again for $120 \mathrm{~s}$. The resulting fractal dimensions correspond well with the values previously determined (Table 2). The strong branching of the second parameter set occurred mainly at the tip of the already existing PEDOT structure, with additional nucleation sites along the strand and even at the gold electrode itself. To investigate the growth dynamics over time, a picture of the video was taken every $20 \mathrm{~s}$, and the grown length and height were measured. Step one (Fig. 4a), indicating the first parameter set, had a constant horizontal growth rate of $9.35 \mu \mathrm{m} / \mathrm{s}$ after an initial nucleation phase of $20 \mathrm{~s}$. In the second phase (Fig. 4b), the spreading of the polymeric brush started with approximately constant rates of $9 \mu \mathrm{m} / \mathrm{s}$ laterally and horizontally (Fig. 4c). Various structures were reliably achieved by simply changing the voltage parameters and did not require any template [2], reduced dimension [7], or change in the ingredients.

\section{Conductive wire}

For determination of the polymer conductivity, a second gold electrode was incorporated into the setup and was shorted with the first WE electrode. The electrodes were placed opposite each other at a distance of $x=1 \mathrm{~mm}$. After 16 min of constant stimulation with $u_{\min }=-2 \mathrm{~V}$ and $\mathrm{DF}=$ $50 \%$, the polymer formed a single connection between the wires (Fig. 5). Consolidation of the polymer between the two wires resulted in a conductive connection with a constant resistance $R=2.8 \mathrm{k} \Omega$, which was confirmed with an electrical impedance spectroscopy measurement. The specific conductance $\sigma=x(R A)^{-1}$ of the PEDOT wire can be estimated as $11.36 \mathrm{~S} \mathrm{~cm}^{-1}$. The area $A$ was approximated based on the average diameter of up to $20 \mu \mathrm{m}$ determined from the scanning electron microscopy images.

This value is in good agreement with values given in other reports [16], in which the PEDOT wires were potentiostatically grown without the risk of overoxidation and were measured in a fully supported electrolyte of TBAP (100 mM).

\section{Migration-driven model}

The substances and the basic frequency of the polymerization were based on a study by Koizumi [6], whereby exact control of the potential across the electrodes was possible. However, the electrolytic reaction system is different from the bipolar condition as in the present case, no cathodic reaction is needed to discharge the floating gold wires. The selected supporting salt TBAP in ACN is 

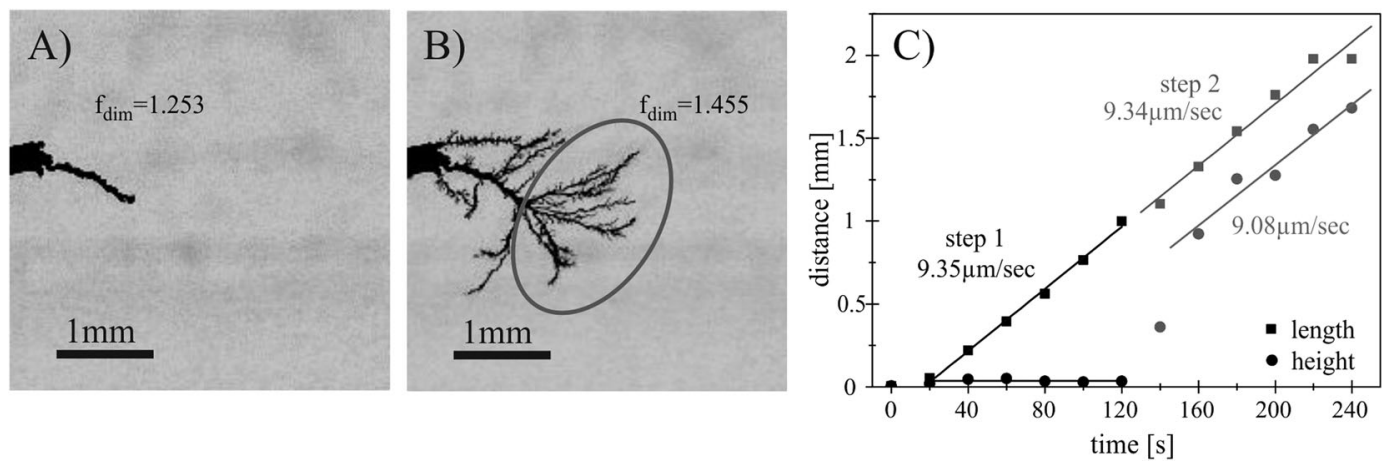

Fig. 4 Two subsequent polymerization parameters for each 120 s. a A deposition with a $6 \mathrm{~V}$ peak-peak $50 \%$ DF followed by $120 \mathrm{~s}$ of electropolymerization at $4 \mathrm{~V}$ with the same DF b. Fractal dimensions are

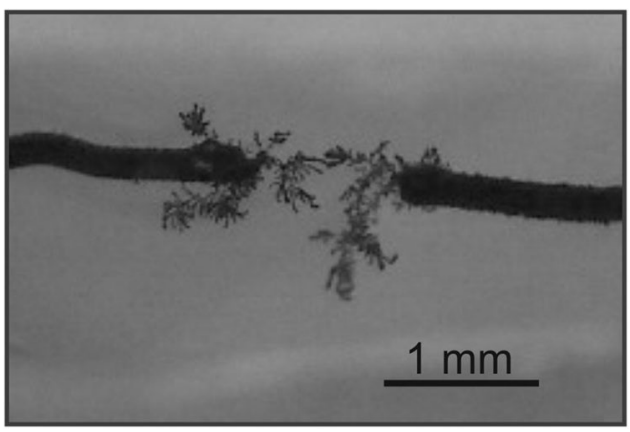

Fig. 5 Conductive connection between two gold electrodes was formed after $16 \mathrm{~min}$ of stimulation with $u_{\min }=-2 \mathrm{~V}$ and $D F=50 \%$. After a stable connection with one single fiber, no further branching or polymerization at the side of the junction was visible. The two uncoated electrodes were initially placed at a distance of $1 \mathrm{~mm}$ from each other

exceptionally low in both cases, playing a major role in the formation of the complex PEDOT structure. The unique effects that occur under such conditions are explained in great detail in the study by Limon-Peterson [9] both theoretically and practically. They modeled chronoamperometric experiments (with the same supporting salt and solvent) using the Nernst-Planck-Poisson equation excluding the assumption of electroneutrality. In summary, with fast switching times $(50-150 \mathrm{~ms})$, the largest potential drop is not expected at the electrode, and the migration of charged ions becomes much more important than in the case of very conductive electrolytes. It can therefore be assumed that only a small proportion of the applied voltage drops near the electrode surface, and thus, it can drive chemical reactions. In the present study, only anodic voltages $u_{\max }$ larger than three volts showed electropolymerization, thereby supporting this theory. It can therefore be assumed that only a fraction (less than half) of the applied voltage dropped directly at the interface. Consequently, the voltage drop across the proximity of the electrode also did not exceed the electrochemical window of the supporting salt given for the entire structure in $\mathbf{a}$ and the red marked region in $\mathbf{b}$. $\mathbf{c}$ The growth rate in the lateral and horizontal directions in $20 \mathrm{~s}$ steps could be linearly approximated after an initial phase $(\sim 20 \mathrm{~s})$

[17]. Most of the voltage dropped via the electrolyte while neglecting the CE given its large size. With strong cathodic pulses, it must also be assumed that the formed PEDOT becomes dedoped and loses part of its conductivity [16]. This occurrence should have a large effect on the growth rate and branching in the first column of Fig. 2a, which is not observed. Especially in panels 4 and 7 (Fig. 2a), the main growth front is still at the tails of the structure. It can be assumed that the short pulse durations and the porous nanostructure of the PEDOT prevent depletion of the $\mathrm{ClO} 4$ - ions. However, the migration of EDOT cations in the bulk electrolyte and the related reorganization of the diffuse layer near the electrode are sufficient to explain the results of the different PEDOT structures.

An important result of this study is that the presented grown structures could not be replicated by using the same waveforms with the Autolab impedance measuring device, although the device is able to supply the required currents. The most obvious explanation is the large difference in the rise times during switching between positive and negative voltages of the two devices used (Agilent $10 \mathrm{~ns} /$ Autolab $10 \mu$ s). It is assumed that a shorter ramp time can have a lower mass transport resistance than an interfacial chargetransfer resistance [18]. In addition, Streeter et al. [10] showed a non-negligible influence of the phase boundary in the nanosecond to microsecond range after voltage switching at low concentrations of supporting salt. The resulting strong difference in the average current during switching seems to be an essential part of the growth process.

The resulting adjustability of the growth behavior can have a great impact on a variety of applications using electrically conductive fractals. On the one hand, it can be applied directly to mature fields such as electrode surface modification [4], neuronal devices [19-21], fractal antennas [22], or solar technology [23]. On the other hand, the adjustable growth patterns may also contribute to more uncommon applications, such as diffraction fractal apertures [24], supercapacitors [25], time-dependent adsorption 
kinetics of fractal surfaces (molecule/gases) $[3,26]$, heat conductors [27], organic electronics towards neuromorphic devices [28], or electronic wire connection [29].

Further studies with simultaneous high-resolution observation of the applied current would lead to a deeper understanding of the growth process. However, a method must be designed to prevent or subtract the back-creeping part of the PEDOT at the wire insulation from the electrode to allow a meaningful interpretation.

\section{Conclusion}

In this work, a stable and controllable growth process of fractal PEDOT structures was successfully achieved by pulsed electropolymerization in low concentrations of supporting salt. Depending on the choice of parameters (voltage amplitude and DF), the conductive polymer grew as a compact film, a branched fractal or a straight fiber without using templates. Although long-lasting strong negative phases of the voltage-driven stimulus led to compact and highly branched PEDOT structures, moredominating positive phases formed a thicker and moredirected polymeric structure. The resulting structures were reliable and can be classified by evaluating the fractal dimension. Even changing the stimulation parameters during one ongoing process led to another expected growth behavior. Thus, it is possible to combine several properties, such as directed growth and strong branching, spatially, and temporally, which further increases the complexity of the structure.

Acknowledgements We thank Dr. Maria Asplund for her advice and the possibility of performing the experiments in her laboratories.

\section{Compliance with ethical standards}

Conflict of interest The author declares that he has no conflict of interest.

Publisher's note: Springer Nature remains neutral with regard to jurisdictional claims in published maps and institutional affiliations.

Open Access This article is licensed under a Creative Commons Attribution 4.0 International License, which permits use, sharing, adaptation, distribution and reproduction in any medium or format, as long as you give appropriate credit to the original author(s) and the source, provide a link to the Creative Commons license, and indicate if changes were made. The images or other third party material in this article are included in the article's Creative Commons license, unless indicated otherwise in a credit line to the material. If material is not included in the article's Creative Commons license and your intended use is not permitted by statutory regulation or exceeds the permitted use, you will need to obtain permission directly from the copyright holder. To view a copy of this license, visit http://creativecommons. org/licenses/by/4.0/.

\section{References}

1. Asplund M, Holst Hvon, Inganäs O. Composite biomolecule/ PEDOT materials for neural electrodes. Biointerphases. 2008;3: 83-93.

2. Richardson-Burns SM, Hendricks JL, Foster B, Povlich LK, Kim D-H, Martin DC. Polymerization of the conducting polymer poly (3,4-ethylenedioxythiophene) (PEDOT) around living neural cells. Biomaterials. 2007;28:1539-52.

3. Martin DC, Malliaras GG. Interfacing electronic and ionic charge transport in bioelectronics. ChemElectroChem. 2016;3:686-8.

4. Cui X, Martin DC. Electrochemical deposition and characterization of poly(3,4-ethylenedioxythiophene) on neural microelectrode arrays. Sens Actuator B Chem. 2003;89:92-102.

5. Kim YH, Sachse C, Machala ML, May C, Müller-Meskamp L, Leo K. Highly conductive PEDOT. PSS electrode with optimized solvent and thermal post-treatment for ITO-free organic solar cells. Adv Funct Mater. 2011;21:1076-81.

6. Koizumi Y, Shida N, Ohira M, Nishiyama H, Tomita I, Inagi S. Electropolymerization on wireless electrodes towards conducting polymer microfibre networks. Nat Commun. 2016;7:10404.

7. Ohira M, Koizumi Y, Nishiyama H, Tomita I, Inagi S. Synthesis of linear PEDOT fibers by AC-bipolar electropolymerization in a micro-space. Polym J. 2016;49:163-7.

8. Watanabe T, Ohira M, Koizumi Y, Nishiyama H, Tomita I, Inagi S. In-plane growth of poly(3,4-ethylenedioxythiophene) films on a substrate surface by bipolar electropolymerization. ACS Macro Lett. 2018;7:551-5.

9. Limon-Petersen JG, Streeter I, Rees NV, Compton RG. Quantitative voltammetry in weakly supported media. effects of the applied overpotential and supporting electrolyte concentration on the one electron oxidation of ferrocene in acetonitrile. J Phys Chem C. 2008;113:333-7.

10. Streeter I, Compton RG. Numerical simulation of potential step chronoamperometry at low concentrations of supporting electrolyte. J Phys Chem C. 2008;112:13716-28.

11. Fernández-Martínez M, Sánchez-Granero MA. Fractal dimension for fractal structures. Topol Appl. 2014;163:93-111.

12. Schindelin J, Arganda-Carreras I, Frise E, Kaynig V, Longair M, Pietzsch T, et al. Fiji: an open-source platform for biologicalimage analysis. Nat Methods. 2012;9:676-82.

13. Kudo K. Fractal analysis of electrical trees. IEEE Trans Dielect Electr Insul. 1998;5:713-27.

14. Cantrell DR, Inayat S, Taflove A, Ruoff RS, Troy JB. Incorporation of the electrode-electrolyte interface into finite-element models of metal microelectrodes. J Neural Eng. 2008;5:54-67.

15. Newman J. Resistance for flow of current to a disk. J Electrochem Soc. 1966;113:501.

16. Morvant MC, Reynolds JR. In situ conductivity studies of poly (3,4-ethylenedioxythiophene). Synth Met. 1998;92:57-61.

17. Buzzeo MC, Hardacre C, Compton RG. Extended electrochemical windows made accessible by room temperature ionic liquid/organic solvent electrolyte systems. ChemPhysChem. 2006;7:176-80.

18. Verbrugge MW, Baker DR. Transient diffusion and migration to a disk electrode. J Phys Chem. 1992;96:4572-80.

19. Wilks SJ, Woolley AJ, Ouyang Liangqi, Martin DC, Otto KJ. In vivo polymerization of poly(3,4-ethylenedioxythiophene) (PEDOT) in rodent cerebral cortex. IEEE Eng Med Biol Soc. 2011;33:5412-5.

20. Thourson SB, Payne CK. Modulation of action potentials using PEDOT:PSS conducting polymer microwires. Sci Rep. 2017;7:10402.

21. Musumeci C, Hutchison JA, Samorì P. Controlling the morphology of conductive PEDOT by in situ electropolymerization. From thin films to nanowires with variable electrical properties. Nanoscale. 2013;5:7756. 
22. Petko JS, Werner DH. Miniature reconfigurable three-dimensional fractal tree antennas. IEEE Trans Antennas Propagat. 2004;52: 1945-56.

23. Xiao Y, Lin J-Y, Tai S-Y, Chou S-W, Yue G, Wu J. Pulse electropolymerization of high performance PEDOT/MWCNT counter electrodes for Pt-free dye-sensitized solar cells. J Mater Chem. 2012;22:19919.

24. Sakurada Y, UOzumi J, Asakura T. Diffraction fields of fractally bounded apertures. Opt Rev. 1994;1:3-7.

25. Ryu KS, Lee Y-G, Hong Y-S, Park YJ, Wu X, Kim KM, et al. Poly(ethylenedioxythiophene) (PEDOT) as polymer electrode in redox supercapacitor. Electrochim Acta. 2004;50:843-7.
26. Nair PR, Alam MA. Dimensionally frustrated diffusion towards fractal adsorbers. Phys Rev Lett. 2007;99:256101.

27. $\mathrm{Xu} \mathrm{P}, \mathrm{Yu} \mathrm{B}$, Yun M, Zou M. Heat conduction in fractal treelike branched networks. Int J Heat Mass Transf. 2006;49: 3746-51.

28. Pecqueur S, Vuillaume D, Alibart F. Perspective: organic electronic materials and devices for neuromorphic engineering. J Appl Phys. 2018;124:151902.

29. Thapa PS, Ackerson BJ, Grischkowsky DR, Flanders BN. Directional growth of metallic and polymeric nanowires. Nanotechnology. 2009;20:235307. 